möglich und deshalb unichtig sind, so muss sein publicistisches Vorgehen auf das strengste verurtheilt werden.

In jener Versammlung behauptete er (nach dem Pensiero), dass, während im ganzen Königreich Italien die Sterblichkeit an Tuberculose nur $2^{1} / 2 \%$ betrage, sie in San Remo unter den Einheimischen auf $18 \%$ gestiegen sei.

Leider sind mir trotz aller Bemühung die officielien statistischen Listen aus Rom nicht zugänglich geworden. 'Trotzlem möchte es nicht schwer sein, den Collegen betreffs seiner Zahlen ad absurdum zu führen.

Denn es ist sicher, dass es kein Land und keine Stadt auf der ganzen Erde giebt, wo die Sterblichkeit an Tuberculose $21 / 9$ auf 100 Einwohner beträgt. Das wäre eine exorbitante Höhe, wie die zum Vergleich herangezogenen Zahlen der beigefügten Tabelle beweisen, die aus mir hier zugänglichen Zusammenstellungen (Eulenburg's Encyklopädie und Statistik der Bewegung der Bevölkerung des Grossherzogthums Baden) entnommen sind.

\begin{tabular}{|c|c|c|c|c|c|}
\hline & $\begin{array}{c}\text { In Preu- } \\
\text { ssen } \\
1875-78\end{array}$ & $\mid \begin{array}{c}\text { In } \\
\text { Bayern } \\
1871-75\end{array}$ & \begin{tabular}{|c|} 
Im Grossh. \\
Baden 1878-87 \\
(Lungen- \\
schwindsucht)
\end{tabular} & $\begin{array}{l}\text { In Frank- } \\
\text { furt a. M. } \\
1851-75\end{array}$ & $\begin{array}{c}\text { In } \operatorname{San} R e m \\
1875-83 \\
\text { (n. Bobone } \\
\text { berechnet) } \\
\end{array}$ \\
\hline $\begin{array}{l}\text { Auf } 1000 \text { Einwohner } \\
\text { starben im Mittel an } \\
\text { Tuberculose..... }\end{array}$ & 3,19 & 2,23 & 2,97 & 3,6 & $\mathbf{2 , 1 5}$ \\
\hline $\begin{array}{l}\text { Auf } 100 \text { Todesfälle } \\
\text { kommen solche an Tu- } \\
\text { berculose } . . . . .\end{array}$ & 12,28 & - & $\begin{array}{c}(1884-87) \\
10,9\end{array}$ & - & 10,4 \\
\hline
\end{tabular}

Man sieht also, dass die Sterblichkeit an Tuberculose nicht pro 100, sondern pro 1000 Einwohner überall zwischen 2 und 3 schwankt.

Als Verhältniss der an 'Tuberculose Gestorbenen zu allen Gestorbenen überhaupt, 'würde aber 2,5 pro 100 eine unmöglch kleine Tuberculosensterblichkeit darstellen. Wahrscheinlich liegt also der Irrthum vor, dass die $2^{1 / 2}$ die Zahl der Todesfälle all 'luberculose auf 1000 le bende Einwohner bedeuten, während $18 \%$ allerdings die Procentzahl der an Tuberculose Gestorbenen sein könnte. Nach Dr. Bobone's Veröffentlichungen ${ }^{1}$ ), der ausser Lungenschwindsucht noch die Intestinaltuberculosa (?) einschliesst, stellt sich diese Zahl für San Remo nur auf 10,4\%, ist also eine relativ geringe. Die den hiesigen Acten sorgfältig entnommenen Zahlen von Bobone sprechen erst recht gegen die aus dem Stegreif gemachten Angaben des Dr. Aicardi, welcher die jener Sterblichkeit sogar noch viel höher als Dr. Macary angiebt, nämlich auf $30 \%$ \%

Dr. A icardi ist nicht Prosector von San Remo, sondern medico necroscopo, d. h. Lei chenbeschauer.

Damit komme ich auf den Werth der Statistik von San Remo über-

\section{Eine Abwehr gegen die Angriffe auf die Riviera speciell San Remo.}

\section{Von Dr. G. Goltz in San Remo-Bad Ems.}

Durch einen Artikel im Pensiero di San Remo, der vielen deutschen politischen und jetzt auch medicinischen Zeitungen zugegangen ist und unter den allarmirendsten Spitzmarken weite Verbreitung gefunden hat, und der auch in No. 16 d. Wochenschr. auszugsweise mitgetheilt wurde, ist ohne jede Berechtigung das Publikum gegen die Rivieracurorte, speciell San Remo, misstrauisch gemacht worden.

Schon im Frühjahr 1889 hatten die deutschen Aerzte in San Rerno sich vereinigt, um die nöthigen Abwehrmaassregeln zu treffen, die der heutige Stand der Wissenschaft erfordert, um eine etwaige Verbreitung speciell der Tuberculose von Seiten der Fremden nach Möglichkeit zu hindern und die Fremdenwohnungen bacillenfrei zu halten.

Es wurden die Vorschläge zur Reinhaltung resp. Desinficirung der Wohnungen und Mobilien in einem Reglement zusammengefasst, dem sich dann im Herbst vorigen Jahres die übrigen hier prakticirenden fremden und einheimischen Aerzte durch Unterschrift anschlossen.

Es wurde dann am 7. Februar a. c. nochinals eine internationale Aerzteversammlung abgebalten und zu dieser auch der Bürgermeister von San Remo eingeladen, weil man wissen wollte, inwieweit die Ortsbehörde durch Polizeiverordnung die Wirthe zur Einhaltung jenes Reglements verpflichten könnte. Niemals aber ist eine Versammlung abgehalten worden, in welcher darüber berathen werden sollte, auf welche Weise der erschrecklichen Zunahue der Schwindsucht unter den Eingeborenen entgegengetreten werden könne." Man wusste bis dahin voll einer solchen Zunahme der Schwindsucht überhaupt nichts.

Da erklärte in dieser Versammlung ein hiesiger italienischer Arzt (Dr. Macary), der ebenfalls jenes Reglement, welches alles praktisch durchführbare verlangte, zum Zeichen seines Einverständnisses unterschrieben hatte, zum grössten Erstaunen der Anwesenden und zum Eutsetzen des Bürgermeisters, man solle den Tuberculösen abrathen, nach San Remo zu kommen, weil sich nach einer von ihm aufgestellten Statistik durch die Anhäufung tuberculöser Kranken hier die Sterblichkeit an Tuberculose unter der eingeborenen Bevölkerung in erschreckender Weise gemehrt habe.

Die eigentlichen Gründe zu diesem Antrage, die anderswo, als in blosser Humanität gegen seine Landsleute zu suchen sein dürften, leuchteten sofort allen ein.

Natürlich wurde unter allgemeinem Protest dieser Antrag zurückgewiesen, durch dessen Annahme nicht nur die.Sanremesen selbst, sondern auch die hier ibrem Beruf und Geschäft nachgehenden Deutschen in ihren berechtigten Interessen auf das empfindlichste geschädigt worden wären.

Hier in der Versammlung mit seinem Antrag durchgefallen, sucht der College durch die Presse seinen Allsichten Verbreitung zu verschaffen.

Wären selbst seine statistischen Angaben richtig, so müsste die Realisirung seines Wunsches doch als eine Chimäre bezeichnet werden, die dem Orte nur schaden, das vermeintliche Uebel aber keineswegs beseitigen oder abschwächen könnte. Da sie aber, wie ich zeigen will, durchaus un - haupt. Die Eintragungen der Todesursachen sind zum Theil ganz unzuverIässig, wenn der Todtenschein nicht vom behandelnden Arzt, sondern vom Leichenbeschauer ausgestellt wird, was sehr häufig geschieht. Denn dieser giebt sich nur selten die Mühe, die wirkliche Todesursache zu constatiren und ist auch oft genıg gar nicht in der Lage, es thun zu können.

Ferner sind die Zahlen in San Remo viel zu klein, um als statistische Unterlage dienen zu können. Handelt es sich (nach Bobone) bei den Einheimischen hier doch nur um im Mittel 28,5 jährliche Todesfälle an Tuberculose! Da machen ein paar Todesfälle mehr oder weniger schon viel aus in Procente übersetzt, ohne irgend welche Bedeutung beanspruchen zu können.

Um aber festzustellen, ob durch den Zuzug fremder Wintergäste sich in der That die Sterblichkeit an Tuberculose gesteigert habe oder nicht, dazu müsste man vor allem wissen, wie gross dieselbe gewesen ist, ehe San Remo ein vielbesuchter Curort gewesen ist. Solche Frmittelungen fehlen und sind auch nicht zu machen, weil erst seit 15 Jahren das Amt des Leichenbeschauers eingerichtet ist und vordem die Todesursachen nicht eingetragen wurden.

Damit ist alles in das subjective Ermessen des einzelnen gestellt. Sollten sich aber in der That in letzter Zeit die Sterblichkeitsverhältnisse an Tuberculose in geringem, noch nicht bewiesemem Grade unter den hiesigen Einwohnern verschlechtert haben, so sind ganz andere Ursachen dafür geltend zu machen, als die Ansteckung durch die fremden Wintergäste.

Einmal sind in den letzten Jahren eine grössere Anzahl Zuzügler aus Italien gekommen, und zwar gerade solche, die als Kranke Sán Remo seines milden Klimas wegen zum Wohnsitz wähltell. Dadurch steigt natürJich die Sterblichkeit.

Dann aber haben sich seit der Anwesenheit der Fremden die socialen Verhältnisse der Einwohner zum grossen Theil geändert. Während früher der grösste Theil derselben seine Campagna bestellte und davon lebte, hat sich natürlich jetzt eine grosse Badeindustrie entwickelt, welche die Leute aus der freien Luft in die geschlossenen Arbeitsräume verjagt und sie dispositionsfähiger machen muss für die Aufnahme von 'Tuberkelbacillen. Es würde damit hier nur so gehen, wie es unter ähnlichen Verhältnissen stets und überall gegangen ist.

Ich glaube durch diese Darlegungen nachgewiesen zu haben, dass die Zahlen, welche beweisen sollen, dass in San Remo unter den Einheimischen die Todesfälle an 'luberculose in erschreckender Weise zugenommen haben, als durchaus unzuverlässige zurückzuweisen sind, und dass es deshalb ganz ungerechtfertigt erscheint, die Fremden vor dem Hierherkommen zu warnen.

Im Gegentheil geht San Remo, dank der Initiative der deutschen Aerzte und dem sachgemässen Verständniss der Orts- und Staatsbehörden, allen anderen Rivieracurorten energisch darin voran, seine Wohnungen, soweit es eben erreichbar ist, rein und frei von Infectionskeimen zu balten. Es ist traurig, dass dieses Bestreben der hiesigen Aerzte jene ungerechtfertigten Folgen haben konnte.

1) Archivio internazionale di Laringologia, Rinologia, Otologia etc. Anno VI. fasc. I. $(1890)$. 